\title{
Turbulence near cyclic fold bifurcations in birhythmic media
}

\author{
Dorjsuren Battogtokh* and John J. Tyson \\ Department of Biology, Virginia Polytechnic and \\ State University Blacksburg, VA 24061-0106
}

(Dated: October 30, 2018)

\begin{abstract}
We show that at the onset of a cyclic fold bifurcation, a birhythmic medium composed of glycolytic oscillators displays turbulent dynamics. By computing the largest Lyapunov exponent, the spatial correlation function, and the average transient lifetime, we classify it as a weak turbulence with transient nature. Virtual heterogeneities generating unstable fast oscillations are the mechanism of the transient turbulence. In the presence of wavenumber instability, unstable oscillations can be reinjected leading to stationary turbulence. We also find similar turbulence in a cell cycle model. These findings suggest that weak turbulence may be universal in biochemical birhythmic media exhibiting cyclic fold bifurcations.

PACS numbers: $\quad$ 05.45.Jn, 05.45.Pq, 82.39.Fk, 82.40.Bj, 89.75.Fb
\end{abstract}

*Electronic address: dbattogt@vt.edu 


\section{INTRODUCTION}

In studies of chemical turbulence in reaction diffusion systems near a Hopf bifurcation, a reduction of the model to the complex Ginzburg Landau equation(CGLE) is very useful 1, 2]. First, it allows to define a parameter set in the model leading to turbulence without carrying out simulations [3]. Secondly, the detailed knowledge of the CGLE's dynamics can be very helpful [4, 5, 6, 7], because mathematical models from different disciplines displaying dynamics near a Hopf bifurcation obey the same qualitative dynamics of the CGLE [8].

However, the CGLE alone is insufficient for a qualitative description of realistic models in a neighborhood of a Hopf bifurcation, when other bifurcations occur nearby [9, 10]. For example, near a supercritical Hopf bifurcation point, another stable limit cycle may exist, so that, depending on initial conditions, oscillations with two different frequencies and amplitudes are possible. Such a situation called birhythmicity is a characteristic feature of a number of well known models in biochemical oscillations [13, 14]. For these systems, the CGLE cannot be used without appropriate modifications. Often, the best way to approach these problems is simulations of the original models [10, 11].

To the best of our knowledge, little is known about turbulence in birhythmic media. Intuitively, in a regime of a strong wavenumber instability, birhythmicity should not be a factor. Therefore, turbulence in homogeneous birhythmic media and in coupled limit cycle oscillators should have similar characteristics. In the absence of wavenumber instability, high frequency oscillations are supposed to suppress slow oscillations and restore uniform oscillations. But at the onset of a cyclic fold $(\mathrm{CF})$ bifurcation in birhythmic media of a biochemical origin, high frequency oscillations may be unstable. Thus, a complete suppression of slow oscillations may not be achieved in these systems. On the contrary, if unstable oscillations emerge persistently, complex spatiotemporal motions are possible.

The goal of this work is to show that near cyclic fold bifurcations in birhythmic media, virtual heterogeneities creating unstable oscillations can lead to a peculiar turbulence, intermittency of small and large amplitude oscillations. We will first compute complex spatiotemporal behavior in a birhythmic medium composed of glycolytic oscillators. By calculating the maximal Lyapunov exponent, the spatial correlation function, and the average transient lifetime, we will provide evidence that this behavior is weak transient turbulence. In the presence of wavenumber instability transient turbulence may become stationary. Math- 
ematically, the instability of the faster oscillations is a result of a CF bifurcation driven by the terms representing enzymatic regulations, suggesting that weak turbulence may be common in biochemical birhythmic media exhibiting CF bifurcations. As further evidence, we demonstrate weak turbulence in a cell cycle model. A biological system where weak turbulence might possibly be found is presented in the closing section.

\section{A BIRHYTHMIC MEDIUM OF GLYCOLYTIC OSCILLATORS}

Let us introduce a birythmic medium composed of glycolytic oscillators,

$$
\begin{aligned}
& \frac{d \alpha}{d t}=\nu+\frac{\sigma_{i} \gamma^{n}}{K^{n}+\gamma^{n}}-\sigma \phi+D_{\alpha} \Delta \alpha \\
& \frac{d \gamma}{d t}=Q \sigma \phi-k_{s} \gamma-\frac{Q \sigma_{i} \gamma^{n}}{K^{n}+\gamma^{n}}+D_{\gamma} \Delta \gamma \\
& \phi=\frac{\alpha(1+\alpha)(1+\gamma)^{2}}{L+(1+\alpha)^{2}(1+\gamma)^{2}}
\end{aligned}
$$

In Eqn. (1-2), $\alpha$ and $\gamma$ represent dimensionless substrate and product concentrations of glycolytic reactions, $K, n, \nu, \sigma_{i}, \sigma, k_{s}, L$ and $Q$ are parameters. For convenience, we assume $Q \equiv 1$ throughout this paper. $D_{\alpha}, D_{\gamma}$ are diffusion constants for the substrate and product. Our units of time and space are sec and $\mathrm{cm}$, respectfully. When $D_{\alpha}=0$, $D_{\gamma}=0$ and $\sigma_{i}=0$, Eqn. (1-2) is called the glycolytic oscillator. The term $\frac{\sigma_{i} \gamma^{n}}{K^{n}+\gamma^{n}}$ represents substrate recycling that drives birhythmicity. Recently, in Ref. 15], Eqn. (1-2) were shown to support multiple wave fronts. Our concern in this paper is a different parameter region where irregular spatiotemporal motions develop.

A phase plane analysis of Eqn. (1-2) shows that the mechanism of birhythmicity is two regions of negative slope in the product nullcline [13]. A convenient way to illustrate birhythmicity is a bifurcation diagram. We used a well known software package, AUTO [16], for bifurcation analysis of the $\operatorname{local} \operatorname{model}\left(D_{\alpha}=D_{\gamma}=0\right.$ in Eqn. (1-2)). Solid lines in Fig. 1 show stable steady states, dashed lines show unstable steady states. Stable limit cycles are shown by filled symbols, unstable limit cycles by open circles. Filled circles represent small amplitude oscillations with high frequencies. Large amplitude oscillations with low frequencies are shown by filled diamonds in Fig. 1. A Hopf bifurcation point $H B$ is located at $\sigma_{i, c r} \approx 1.282$. There are two CF bifurcations in Fig. 1, where stable limit cycles are 
replaced by unstable ones. Between these two CF points, which occur at $\sigma_{i, C F^{1}} \approx 1.77$ and $\sigma_{i, C F^{2}} \approx 1.83$, two stable limit cycles coexist. Therefore, depending on initial conditions, one of the limit cycles will be selected in simulations of the glycolytic oscillator with substrate recycling.

A general mechanism of turbulence in oscillatory reaction diffusion systems is wavenumber instability, i.e., instability of uniform oscillations against phase-like fluctuations [1]. In Eqn. (1-2), there are two different uniform oscillations that might undergo wavenumber instability. We want to provide evidence that these oscillations are stable against phase-like fluctuations for the parameters in Fig. 1. For the fast, uniform oscillations which originate from the Hopf bifurcation point shown by filled circles in Fig. 1, the stability condition can be obtained by reducing Eqn. (1-2) to the CGLE,

$$
\dot{A}=\left(1+i c_{0}\right) A-\left(1+i c_{2}\right)|A|^{2} A+\left(1+i c_{1}\right) \Delta A
$$

In Eqn. (3) $A$ is the complex amplitude, and $\omega, \beta, \gamma$ are real parameters. The CGLE has a uniform oscillatory solution, $A=\exp \left(i\left(c_{0}-c_{2}\right) t\right)$, that is stable if the condition $1+c_{1} c_{2}>0$ holds. In the appendix, we calculated $c_{0}, c_{1}$ and $c_{2}$ corresponding to Eqn. (1-2). Our results show that the uniform oscillations are stable for the parameters used in Fig. 1, for any $D_{\alpha}>0$ and $D_{\gamma}>0$. For $D_{\alpha}=D_{\gamma}$ we find that $c_{1}=0$. Hence, the parameter region we are interested in is deep inside the Benjamin-Feir stability region given by $1+c_{1} c_{2}>0$. Although the CGLE is valid only near the $H B$ point, it is likely that the uniform oscillations will remain stable until the the next bifurcation in the system, i.e., $C F^{1}$ in Fig. 1 [8]. Next, consider the uniform oscillations with low frequencies. Unlike the case of fast oscillations, no analytic approach is available in this case. Note that oscillations shown by filled circles and diamonds in Fig. 1 occur at the same parameters. Therefore, it is rather unlikely that the slow oscillations undergo wavenumber instability, contrary to the fast ones. Thus, we can assume that uniform, slow oscillations are also stable.

It is known that strong perturbations can switch oscillations from one stable orbit to another in the glycolyctic model with substrate recycling [13]. Therefore, even if both uniform oscillations in Eqn. (1-2) are stable against wavenumber instability, strong perturbations can excite the system by switching the oscillations. This kind of excitability, however, will

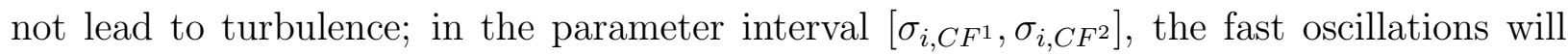
suppress the slow ones as time progresses. But for $\left|\sigma_{i}-\sigma_{i, C F^{1}}\right| \ll 1$ where the fast oscil- 
lations become unstable, it is apparent that a complete suppression of slow oscillations is impossible. Here, because of complex interactions between stable, slow and unstable, fast oscillations, interesting spatiotemporal dynamics might develop. Therefore, we carried out a detailed numerical study in the neighborhood of $C F^{1}$.

\section{WEAK TURBULENCE IN A BIRHYTHMIC MEDIUM OF GLYCOLYTIC OSCILLATORS}

For numerical integrations of Eqn. (1-2) in one spatial dimension, we used the fourth order Runge-Kutta method. Diffusion terms were approximated by the finite difference method. Numerical parameters are $\delta x=0.005 \mathrm{~cm}, \delta t=0.05 \mathrm{~s}$. The system size is defined as $l=N \delta x$, where $N$ is the number of spatial grid points. In this paper we present results for periodic boundary conditions, but we also tested the main results with no-flux boundary conditions. We also tested selected examples with smaller values of $\delta x$ and $\delta t$ for fixed $l$. Our simulations show that Eqn. (1-2) are sensitive to initial conditions. By choosing initial conditions as small perturbations of uniform, slow oscillations with large amplitudes, we found that these oscillations are stable for $\sigma_{i}<\sigma_{i, C F^{2}}$. But, near and to the left of $C F^{1}$, uniform, fast oscillations with small amplitudes are found to be unstable. They spiral out from unstable orbits towards the orbit of stable, large amplitude oscillations. For strong perturbations near the $C F^{1}$ bifurcation point, we found spatiotemporal irregular motions in Eqn. (1-2).

Fig. 2 shows a gray scale plot of spatiotemporal dynamics in Eqn. (1-2). Oscillations between the white and black colors show large amplitude oscillations displayed by $\gamma(x, t)$. There are also oscillations with higher frequency and smaller amplitude in Fig. 2. Because the latter ones are unstable, they can not suppress large amplitude domains. Although uniform, large amplitude oscillations are stable against small fluctuations, phase slips created by strong initial perturbations cannot be eliminated as time progresses. As a result, spatially nonuniform distributions of concentrations are seen at given time moments, Fig. 3. On the

phase plane, these nonuniform distributions generate motions attracted by unstable orbits around the inner cycle shown in Fig. 4. We found that such unstable orbits act as a weak, virtual heterogeneity emerging randomly. They cannot entrain the bulk oscillations, but in their presence, phase slips cannot be eliminated. Instead, persistent spatiotemporal irregular 
motions develop.

To characterize the irregular motions in Fig. 2, we calculated the maximum Lyapunov exponent $\lambda_{\text {lyap }}^{\max }$ in $2 N$ dimensional phase space [17]. First we made a very long run of Eqn. (1-2) to confirm that the turbulence is stationary. Then, by using the same initial conditions, we simulated Eqn. (1-2) and its linear system for computation of $\lambda_{\text {lyap }}^{\max }$ for $T_{1}=2 \cdot 10^{5} \mathrm{~s}$. We found that the largest Lyaponov exponent is positive and small, $\lambda_{\text {lyap }}^{\text {max }} \approx 2 \cdot 10^{-3}$. We also calculated a two-point correlation function, $C(x)=<\gamma\left(x_{0}, t\right) \gamma\left(x_{0}+x, t\right)>$, where $<. .>$ stands for an average over space and time [18]. Fig. 5 shows that $C(x) \approx$ const at small values of $x$, indicating strong local coupling and an absence of short waves. A power-law decay of the correlation function at intermediate values of $x$ implies the presence of chaotic motions. We found that the slope is $\kappa \approx-0.15$. We also found no significant variations of $\kappa$ and $\lambda_{\text {lyap }}^{\text {max }}$ with changes of $\sigma_{i}$ and $l$. The small values of $\kappa$ and $\lambda_{\text {lyap }}^{\max }>0$ suggest that spatiotemporal irregular motions shown in Fig. 2-4 can be characterized as a weak turbulence.

We found that in Eqn. (1-2), stationary irregular motions can develop only for certain initial conditions and system sizes. In simulations with different initial conditions and system sizes, we observed sudden collapses of turbulent dynamics. Collapse of turbulence in Eqn. (1-2) means a complete suppression of small amplitude oscillations. Thus, we defined the transient lifetime of turbulence $t_{p}$ as the time interval from initial conditions to the moment when all oscillators come within a distance $d$ of the orbit of stable, slow, large amplitude oscillations. In our simulations we used $d=0.03$. Following Ref. [12], we plot an average transient lifetime $t_{p}$ versus the system size $l$ in Fig. 6. Here, each filled circles is an average of 20 simulations with different initial conditions. Fig. 6 shows that, as the system size increase, $t_{p}$ grows exponentially.

For some initial conditions, when $l$ is close to $2 \mathrm{~cm}$, the turbulent solution does not collapse. The inset in Fig. 6 shows the number of cases, among 20 different simulations, when a collapse of turbulence has not occurred by $T=10^{6} \mathrm{~s}$. (These cases were not included in calculations of the filled circles in Fig. 6.) We continued two cases in the inset (at $l=1.75 \mathrm{~cm})$ up to $T=10^{8} \mathrm{~s}$ and did not observe a collapse of motions near the inner cycle in Fig. 4.

Numerical experiments indicate that if virtual heterogeneities reside sufficiently far from each other, a stationary pattern is possible in the interval $\sigma_{i} \in[1.055,1.075]$. Fig. 7 gives 
an example of such a pattern. Here virtual heterogeneities are located from each other by distances between $0.5 \mathrm{~cm}$ and $1 \mathrm{~cm}$. Note that these quasi-periodic structures are not related to a Turing instability, which emerges due to differences in diffusion coefficients. Unstable oscillations at the onset of a CF bifurcation are the instabilities leading to these structures. The cellular structures in Fig. 7 are breathing because of the unstable oscillations. Numerical results show that as the system size increases, the cells breath coherently.

A collapse of turbulence can be prevented if there is a reinjection mechanism for the unstable oscillations generated by the virtual heterogeneities. Naturally, wavenumber instability can be such a mechanism. Using our calculations in the appendix, we simulated Eqn. (1-2) for parameters when the corresponding CGLE displays a wavenumber instability and found that small amplitude oscillations exhibit phase instability near Hopf bifurcation. For $\left|\sigma-\sigma_{i, C F^{1}}\right| \ll 1$ we found stationary weak turbulence.

\section{WEAK TURBULENCE IN A CELL CYCLE MODEL}

In section III, we demonstrated that the $C F^{1}$ bifurcation point is crucial for turbulence in Eqn. (1-2). Mathematically, the term representing substrate recycling drives CF bifurcations. In models of biochemical oscillations, terms representing enzymatic activities naturally arise. As an enzyme can quickly switch from being active to inactive and back again, ideal conditions for CF bifurcations exist in these models. Therefore, other biochemical reaction diffusion models may also display the weak turbulence discussed in the previous section. As an example, consider a three variable model of the budding yeast cell cycle,

$$
\begin{aligned}
& \frac{d X}{d t}=m\left(k_{1}+k_{2} T\right)-\left(k_{3}+k_{4} Y+k_{5} Z\right) X+D_{X} \Delta X, \\
& \frac{d Y}{d t}=\frac{\left(k_{6}+k_{7} Z\right)(1-Y)}{J_{1}+1-Y}-\frac{\left(k_{8} m+k_{9} X\right) Y}{J_{1}+Y}+D_{Y} \Delta Y, \\
& \frac{d Z}{d t}=\left(k_{10}+k_{11} X\right)-k_{12} Z+D_{Z} \Delta Z, \\
& T=G\left(X, P, J_{2}, J_{2}\right), \\
& G(a, b, c, d)=\frac{2 a d}{b-a+b c+a d+\sqrt{(b-a+b c+a d)^{2}-4 a d(b-a)}},
\end{aligned}
$$

where the transcription factor $T$ for $X$ is given by the Goldbeter-Koshland function $G$ [13]. $X, Y, Z$ are dimensionless variables and $m$ is a dimensionless parameter. Our units of time 
and space are min and $\mathrm{cm}$, respectfully.

When $D_{X}=D_{Y}=D_{Z}=0$, Eqn. (4-8) are the reduced version of a budding yeast cell cycle model [19]. Here, $X$ represents the concentration of cyclin-dependent protein kinase(CDK), $Y$ and $Z$ are concentrations of two different anaphase promoting complexes(APC), APC/Cdh1 and APC/Cdc20 respectively. In Eqn. (4-5), $m$ represents the cell's mass, which will be used as a primary bifurcation parameter. Eqn. (4-8) display CF bifurcations as shown in Fig. 8. For small $m$, Eqn. (4-8) also display saddle node bifurcations, a feature universal in cell cycle models [14, 19]. Here, our concern is the neighborhood of $C F^{1}$ in Fig. 8.

There are no experimental measurements of diffusion coefficients for CDK, and APC factors. But it is known that diffusion coefficients of average sized proteins in cytoplasm are

order of $10^{-4} \frac{\mathrm{cm}^{2}}{\mathrm{~min}}$ or smaller [20, 21]. As our goal is a demonstration of weak turbulence in a representative model of biochemical oscillations, we choose $D_{X}$ and $D_{Z}$ arbitrarily subject to this upper bond. For simplicity, we assume $D_{Z}=0$.

For simulations of Eqn. (4-8) we used the same method as in the previous section with $\delta t=0.05 \mathrm{~min}, \delta x=0.005 \mathrm{~cm}$. We found numerically that for strong perturbations, Eqn. (4-8) display a weak turbulence, Fig. 9. Typically, for $D_{X} \leq D_{Y}$, we found transient, weak turbulence. When $D_{X}<<D_{Y}$, numerical experiments lead to stationary turbulence. For instance, we simulated Eqn. (4-8) up to $T=10^{7} \mathrm{~min}$ for $m=3, D_{X}=6 \cdot 10^{-7} \frac{\mathrm{cm}^{2}}{\min }$, $D_{Y}=10^{-4} \frac{\mathrm{cm}^{2}}{\min }, D_{Z}=0$ and $l=1.28 \mathrm{~cm}$ and found stationary turbulence for a number of different initial conditions.

\section{DISCUSSION}

We have shown in this paper that two representative mathematical models of biochemical oscillations exhibiting birhythmicity, glycolytic and cell cycle models, display weak turbulence, intermiitency of large and small amplitude oscillations. We revealed that unstable oscillations near cyclic fold bifurcations are the mechanism of transient turbulence in birhythmic media. In the presence of wavenumber instability, weak turbulence is stationary.

Recently, Stich et. al. [22, 23] proposed an amplitude model for birhythmic media. An interesting question is whether the weak turbulence we discussed in this paper can be found in their model? First, let us mention two important differences between our models 
and the amplitude model of birhythmic media. In our case, a cyclic fold bifurcation is crucial for turbulence, but the amplitude model describes a pitch-fork bifurcation of limit cycles. Secondly, both fast and slow oscillations in the amplitude equation are smooth, but in our case, slow oscillations are strongly anharmonic. Besides these differences, it is well known that if phase slips develop, the CGLE equation generates defects [24]. Thus, these facts indicate that instead of intermittency of small and large amplitude oscillations, defect turbulence is likely in the amplitude model of birhythmic media.

To date, there are no experimental evidences of weak turbulence in glycolysis or in the cell cycle. Our results are pure theoretical predictions of mathematical models. The system sizes we simulated are much larger than the typical size of an yeast cell $\left(10^{-3} \mathrm{~cm}\right)$. Therefore, weak turbulence is not expected in yeasts. Interestingly, some slime molds grow as syncytial plasmodia (many nuclei in a common cytoplasmic pool) that are many times larger than

a typical yeast cell; cells $15 \mathrm{~cm}$ in diameter can be grown in the laboratory [25]. Waves of nuclear division are observed in these multinucleate plasmodia [26, 27], and, as we have shown it is possible that these waves exhibit weak turbulence. Note that weak turbulence in the cell cycle would mean irregular oscillations of CDK. But for a normal cell cycle, large amplitude oscillations of CDK are essential; CDK activity must drop below a certain threshold for nuclei to exit mitosis and divide. Therefore, hypothetically, weak turbulence in syncytial plasmodia might lead to mitotic arrest of certain nuclei in the plasmodium.

A more quantitative characterization of wavenumber instability of unstable oscillations at the onset of a cyclic fold bifurcation, as well as simulations in two spatial dimensions 28] are problems in the future.

\section{APPENDIX A: COEFFICIENTS OF THE CGLE FOR A GLYCOLYTIC MODEL WITH SUBSTRATE INHIBITION}

In this appendix, following standard procedures in Ref. 1], we will calculate coefficients of CGLE for the glycolytic model. For a convenience we assume $Q \equiv 1$ in Eqn. (1-3). First, let us find uniform steady state solutions $\alpha_{0}$ and $\gamma_{0}$,

$$
\begin{gathered}
\gamma_{0}=\mu / k_{s} \\
\alpha_{0}=\frac{K^{4}(-2 \mu+\sigma)+\gamma_{0}^{4}\left(-2\left(\mu+\sigma_{i}\right)+\sigma\right)}{\tilde{c}}-
\end{gathered}
$$




$$
-\frac{\sqrt{-4 \tilde{a}^{2}+4 L \sigma \tilde{a} \tilde{b}+\left(1+\gamma_{0}\right)^{2} \sigma^{2} \tilde{b}^{2}}}{\left(1+\gamma_{0}\right) \tilde{c}}
$$

where, $\tilde{a}=K^{4} \mu+\gamma_{0}^{4}\left(\mu+\sigma_{i}\right), \tilde{b}=\left(K^{4}+\gamma_{0}^{4}\right), \tilde{c}=2\left(K^{4}(\mu-\sigma)+\gamma_{0}^{4}\left(\mu+\sigma_{i}-\sigma\right)\right)$. Next we perform a linear stability analysis of $\left(\alpha_{0}, \gamma_{0}\right)$ against small fluctuations $\delta \alpha, \delta \gamma \propto \exp (i q x+i \lambda t)$. At the critical wavenumber $q_{c r}=0$, we obtain a characteristic equation,

$$
\lambda^{2}+\left(a_{1}+a_{2}+k_{s}\right) \lambda+a_{1} k_{s}=0
$$

In Eqn. (A3), $a_{1}$ and $a_{2}$ are given by

$$
\begin{gathered}
a_{1}=\frac{\sigma\left(1+\gamma_{0}\right)^{2}\left(L+2 L \alpha_{0}+\left(1+\alpha_{0}\right)^{2}\left(1+\gamma_{0}\right)^{2}\right)}{\left(L+\left(1+\alpha_{0}\right)^{2}\left(1+\gamma_{0}\right)^{2}\right)^{2}}, \\
a_{2}=\frac{4 K^{4} \gamma_{0}^{3} \sigma_{i}}{\left(K^{4}+\gamma_{0}^{4}\right)^{2}}-\frac{2 \sigma L \alpha_{0}\left(1+\alpha_{0}\right)\left(1+\gamma_{0}\right)}{\left(L+\left(1+\alpha_{0}\right)^{2}\left(1+\gamma_{0}\right)^{2}\right)^{2}} .
\end{gathered}
$$

Let us define such a critical value for the bifurcation parameter $\sigma_{i}=\sigma_{i, c r}$ that

$$
a_{1}+a_{2}+k_{s} \equiv 0
$$

Eqn. (A6) is the condition for a Hopf bifurcation; the characteristic equation has pure imaginary solutions, $\lambda_{0}= \pm i \sqrt{a_{1} k_{s}}$.

Let $\mu$ be defined by $\mu=\frac{\sigma_{i}-\sigma_{i, c r}}{\sigma_{i, c r}}$. We develop the Jacobian matrix $L$ of Eqn. (1-3) in powers of $\mu$,

$$
L=L_{0}+\mu L_{1}+\ldots
$$

At $\mu=0$ the Jacobian is given by

$$
L_{0}=\left|\begin{array}{cc}
-a_{1} & a_{2} \\
a_{1} & -k_{s}-a_{2} .
\end{array}\right| .
$$

We find the right $\mathbf{u}_{\mathbf{0}}$ and left $\mathbf{u}_{\mathbf{0}}{ }^{*}$ eigenvectors of $L_{0}$ corresponding to $\lambda_{0}$,

$$
\begin{gathered}
\mathbf{u}_{\mathbf{0}}=\left(\begin{array}{c}
-1+i \sqrt{\frac{k_{s}}{a_{1}}} \\
1
\end{array}\right), \\
\mathbf{u}_{\mathbf{0}}{ }^{*}=\frac{1}{2}\left(-i \sqrt{\frac{a_{1}}{k_{s}}}, 1-i \sqrt{\frac{a_{1}}{k_{s}}}\right) .
\end{gathered}
$$


We find further,

$$
L_{1}=\frac{4 K^{4} \gamma_{0}^{3} \sigma_{i, c r}}{\left(K^{4}+\gamma_{0}^{4}\right)^{2}}\left|\begin{array}{cc}
0 & -1 \\
0 & 1
\end{array}\right|
$$

Let us first find $c_{0}$ in the CGLE. It is given by $c_{0}=\frac{\operatorname{Im} \lambda_{1}}{\operatorname{Re} \lambda_{1}}$, where

$$
\lambda_{1}=\mathbf{u}_{\mathbf{0}}{ }^{*} L_{1} \mathbf{u}_{\mathbf{0}}=\frac{2 K^{4} \gamma_{0}^{3}}{\left(K^{4}+\gamma_{0}^{4}\right)^{2}} \sigma_{i, c r} .
$$

We see that $\lambda_{1}$ is pure real, therefore, $c_{0}=0$. Now following again [1], we find $c_{1}$,

$$
\begin{gathered}
D=\left|\begin{array}{cc}
D_{\alpha} & 0 \\
0 & D_{\gamma}
\end{array}\right|, \\
d^{\prime}+i d^{\prime \prime}=\mathbf{u}_{\mathbf{0}}{ }^{*} D \mathbf{u}_{\mathbf{0}}, \\
c_{1}=d^{\prime \prime} / d^{\prime}=\sqrt{\frac{a_{1}}{k_{s}}}\left(\frac{D_{\alpha}-D_{\gamma}}{D_{\alpha}+D_{\gamma}}\right) .
\end{gathered}
$$

Calculation of $c_{2}$ is a little more tedious. We need to find [1],

$$
\begin{gathered}
\mathbf{V}_{+}=\overline{\mathbf{V}}_{-}=-\left(L_{0}-2 \lambda_{0}\right)^{-1} \mathbf{M}_{0} \mathbf{u}_{0} \mathbf{u}_{0}, \\
\mathbf{V}_{0}=-2 L_{0}{ }^{-1} \mathbf{M}_{0} \mathbf{u}_{0} \overline{\mathbf{u}}_{0}, \\
g=g^{\prime}+i g^{\prime \prime}=-2 \mathbf{u}_{\mathbf{0}}{ }^{*} \mathbf{M}_{0} \mathbf{u}_{0} \mathbf{V}_{0}-2 \mathbf{u}_{\mathbf{0}}{ }^{*} \mathbf{M}_{0} \overline{\mathbf{u}}_{0} \mathbf{V} \\
-3 \mathbf{u}_{\mathbf{0}}{ }^{*} \mathbf{N}_{0} \mathbf{u}_{\mathbf{0}}{ }^{*} \mathbf{u}_{\mathbf{0}}{ }^{*} \overline{\mathbf{u}}_{0} .
\end{gathered}
$$

Parameter $c_{2}$ in the CGLE is given by a formula, $c_{2}=g^{\prime \prime} / g^{\prime}$. We find that $c_{2}=\frac{\tilde{g}^{\prime \prime}}{\tilde{g}^{\prime}}$, where

$$
\begin{gathered}
\tilde{g}^{\prime}=-3 k_{s}\left[k_{s} m_{\alpha^{2}}\left(2 m_{\alpha^{2}}-m_{\alpha \gamma}\right)+a_{1}\left(\left(2 m_{\alpha^{2}}-m_{\alpha \gamma}\right)\left(m_{\alpha^{2}}-m_{\alpha, \gamma}+m_{\gamma^{2}}\right)\right.\right. \\
\left.\left.-k_{s}\left(n_{\alpha^{2} \gamma}-3 n_{\alpha^{3}}\right)\right)+3 a_{1}^{2}\left(n_{\alpha \gamma^{2}}-n_{\alpha^{2} \gamma}+n_{\alpha^{3}}-n_{\gamma^{3}}\right)\right], \\
\tilde{g}^{\prime \prime}=\sqrt{\frac{k_{s}}{a_{1}}}\left[10 k_{s}^{2} m_{\alpha^{2}}^{2}+a_{1} k_{s}\left(14 m_{\alpha^{2}}^{2}-14 m_{\alpha^{2}} m_{\alpha, \gamma}+m_{\alpha \gamma}{ }^{2}+10 m_{\alpha^{2}} m_{\gamma^{2}}+9 k_{s} n_{\alpha^{3}}\right)+\right. \\
\left.+a_{1}^{2}\left(4\left(m_{\alpha^{2}}-m_{\alpha, \gamma}+m_{\gamma^{2}}\right)^{2}+3 k_{s}\left(n_{\alpha \gamma^{2}}-2 n_{\alpha^{2} \gamma}+3 n_{\alpha^{3}}\right)\right)\right] .
\end{gathered}
$$

In the above expressions, $m_{\alpha^{2}}=\left(\frac{\partial^{2} \phi(\alpha, \gamma)}{\partial \alpha^{2}}\right)_{\alpha_{0}, \gamma_{0}}, m_{\alpha \gamma}=\left(\frac{\partial^{2} \phi(\alpha, \gamma)}{\partial \alpha \partial \gamma}\right)_{\alpha_{0}, \gamma_{0}}, m_{\gamma^{2}}=$ $\frac{2\left(3 K^{8} \gamma_{0}^{2}-5 K^{4} \gamma_{0}^{6}\right) \sigma_{i, c r}}{\left(K^{4}+\gamma_{0}^{4}\right)^{3}}-\left(\frac{\partial^{2} \phi(\alpha, \gamma)}{\partial \gamma^{2}}\right)_{\alpha_{0}, \gamma_{0}}, \quad n_{\alpha^{3}}=\left(\frac{\partial^{3} \phi(\alpha, \gamma)}{\partial \alpha^{3}}\right)_{\alpha_{0}, \gamma_{0}}, n_{\alpha^{2} \gamma}=\left(\frac{\partial^{3} \phi(\alpha, \gamma)}{\partial \alpha^{2} \partial \gamma}\right)_{\alpha_{0}, \gamma_{0}}, n_{\alpha \gamma^{2}}=$ $\left(\frac{\partial^{3} \phi(\alpha, \gamma)}{\partial \alpha \partial \gamma^{2}}\right)_{\alpha_{0}, \gamma_{0}}, n_{\gamma^{3}}=\left(\frac{\partial^{3} \phi(\alpha, \gamma)}{\partial \gamma^{3}}\right)_{\alpha_{0}, \gamma_{0}}$. To save space we do not present here cumbersome expressions for these derivatives. 
For parameters in Fig. 1 we find that $\sigma_{i, c r}=1.282$ and $c_{2} \approx 2.21$. From Eqn. (A15) we see that if $D_{\alpha}=D_{\gamma}, c_{1}=0$. Therefore, $1+c_{1} c_{2}>0$ for parameters used in this paper. If $D_{\alpha}=0$, Eqn. (A15) gives the minimal value, $c_{1}=-0.47$. In this case $1+c_{1} c_{2} \approx-0.03$, therefore, wavenumber instability is possible. However, turbulence must be weak as the parameters are very close to the stability condition $1+c_{1} c_{2}>0$ [2]. A stronger wavenumber instability is possible, for example, for $\mu=0.28 \mathrm{~s}^{-1}, K=12, D_{\alpha}=5 \cdot 10^{-7} \frac{\mathrm{cm}^{2}}{\mathrm{~s}}, D_{\gamma}=1 \cdot 10^{-5} \frac{\mathrm{cm}^{2}}{\mathrm{~s}}$ and other papameters are the same as in Fig. 1 . In this case, we find that $\sigma_{i, c r} \approx 1.095$ and $1+c_{1} c_{2}=-0.416$

[1] Y. Kuramoto, Chemical Oscillations, Waves and Turbulence, Springer-Verlag, Berlin, 1984.

[2] M. C. Cross and P. C. Hohenber, Rev. Mod. Phys. 65, 851 (1993).

[3] Y. Kuramoto, D. Battogtokh, and H. Nakao, Phys. Rev. Lett. 81, 3543 (1998).

[4] K. Nozaki and N. Bekki, Phys. Lett. A 110, 133 (1985).

[5] B. I. Shraiman, A. Pumir, W. van. Saarlos, P. C. Hohenberg, H. Chate, and M. Holen, Physica D 57, 241 (1992).

[6] H. Sakaguchi, Progr. Theor. Phys. 84, 792 (1990).

[7] I. S. Aronson, L. Kramer, Rev. Mod. Phys. 74, 99 ( 2002).

[8] H. Mori and Y. Kuramoto, Dissipative Structures and Chaos, Springer-Tokyo, 1998.

[9] M. Ipsen, L. Kramer, P. G. Sorensen, Physics Reports 337, 193(2000).

[10] J. H. Merkin, V. Petrov, S. K. Scott and K. Showalter, Phys. Rev. Lett. 76, 546(1996).

[11] Y. Nishiura and D. Ueyama, Physica D 150, 137(2001).

[12] R. Wackerbauer and K. Showalter, Phys. Rev. Lett. 91, 174103, 2003.

[13] A. Goldbeter, Biochemical Oscillations and Cellular Rhythms. The Molecular Bases of Periodic and Chaotic Behavior, Cambridge University Press, UK, 1996.

[14] M. T. Borisuk and J. J. Tyson, Journ. Theor. Biol. 195, 69 (2000).

[15] C. Perez-Iratxeta, J. Halloy, F. Moran, J. L. Martel, A. Goldbeter, Biophys. Chem. 74, 197 (1998).

[16] E. J. Doedel, A. R. Champneys, T. F. Fairgrieve, Y. A. Kuznetsov, B. Sandstede, X. Wang, Auto97, Continuation and Bifurcation Software for Ordinary Differential Equations(with Hom Cont), 1997. 
[17] A. Wolf, J. B. Swift, H. L. Swinney and J. Vastano, Physica D 16, 285 (1985).

[18] D. Battogtokh, Phys. Rev. E 66, 066202 (2002).

[19] K. Chen, A. Csikasz-Nagy, B. Georffy, J. Val, B. Novak, J. J. Tyson, Mol. Biol. Cell 11, 369 (2000); D. Battogtokh and J. J. Tyson, preprint (2004).

[20] J. W. Wojcieszyn, R. A. Schlegel, E. S. Wu, K. A. Jacobson, PNAS USA 78, 4407 (1981).

[21] G. Zubay, Biochemistry, Addison Wesley, Reading MA, 1983.

[22] M Stich, M. Ipsen and A. S. Mikhailov, Phys. Rev. Lett., 86, 4406 (2001).

[23] M. Stich, M. Ipsen, A. S. Mikhailov, Physica D 171, 19 (2002).

[24] H. Chate, Nonlinearity 7, 185, (1994).

[25] J. Mohberg and H. P. Rusch, Journal of Bacteriology 97, 1411 (1969).

[26] E. F. Haskins, Film E 2000 Goetingen. Inst. Wiss. Film 1974.

[27] B. Novak and J. J. Tyson, Journ. Theor. Biol. 165, 101 (1993).

[28] Our preliminary results show existence of the weak turbulence in dimension two.

\section{LIST OF FIGURES}

FIG. 1. A bifurcation diagram of Eqn. (1-3). HB marks a Hopf bifurcation point, $\mathrm{CF}^{1,2}$

mark cyclic fold bifurcations. Parameters are: $\nu=0.25 \mathrm{~s}^{-1}, n=4, K=11.5, \sigma=11 \mathrm{~s}^{-1}$, $k_{s}=0.05 \mathrm{~s}^{-1}, L=3400000$.

FIG. 2. Space-time pattern of $\gamma$ in a weak turbulent regime of Eqn. (1-2). The space and time spans are $l=1.75 \mathrm{~cm}$ and $T=5 \cdot 10^{3} \mathrm{~s}$. The pattern was obtained by recording $\gamma(x)$ with a time interval $\tau=5 \mathrm{~s} . D_{\alpha}=D_{\gamma}=1 \cdot 10^{-5} \frac{\mathrm{cm}^{2}}{\mathrm{~s}}$ and $\sigma_{i}=1.065 \mathrm{~s}^{-1}$. Other parameters are the same as in Fig. 1.

FIG. 3. Snapshots of spatial distributions of $\alpha$ at two different time moments. Parameters are the same as in Fig. 2.

FIG. 4. A phase plane view. The outer cycle shows the orbit of stable uniform oscillations with a period $\tau=300$ s. The inner cycle shows the orbit of small amplitude, fast oscillations with a period $\tau=290 \mathrm{~s}$ at $\sigma_{i}=1.08 \mathrm{~s}^{-1}$. With the decrease of $\sigma_{i}$, the inner cycle disappears, but it still can attract neighboring trajectories creating a virtual, chaotic heterogeneity in Eqn. (1-2). The solid lines show oscillator distributions at two different time moments. Parameters are the same as in Fig. 2. 
FIG. 5. A log-log plot of the spatial correlation function. Parameters are the same as in Fig. 2.

FIG. 6 Average transient lifetime versus the system size. The inset shows the cases when a collapse of turbulence has not occurred by $T=10^{6} \mathrm{~s}$. Parameters are the same as in Fig. 2 .

FIG. 7. Breathing periodic structures. $l=3.5 \mathrm{~cm}$, other parameters, as well as the time and space spans are the same as in Fig. 2.

FIG. 8. Bifurcation diagram of a cell cycle model. Rate constants $k_{i}$ are in units min $^{-1}$, $k_{1}=0.002, k_{2}=0.053, k_{3}=0.01, k_{4}=2, k_{5}=0.05, k_{6}=0.04, k_{7}=1.5, k_{8}=0.19$, $k_{9}=0.64, k_{10}=0.005, k_{11}=0.07, k_{12}=0.08$. Other parameters are $P=0.15, J_{1}=0.05$, and $J_{2}=0.01, l=1.28 \mathrm{~cm}, D_{X}=6 \cdot 10^{-7} \frac{\mathrm{cm}^{2}}{\min }, D_{Y}=10^{-4} \frac{\mathrm{cm}^{2}}{\min }$ and $D_{Z}=0$.

FIG. 9. Turbulence in a cell cycle model. Space time plot of $Y$ field in Eqn. (4-8). The space and time spans are $L=1.28 \mathrm{~cm}$ and $T=2500 \mathrm{~min}$. The pattern was obtained by recording $Y(x)$ with a time interval $\tau=5 \mathrm{~min}$. 


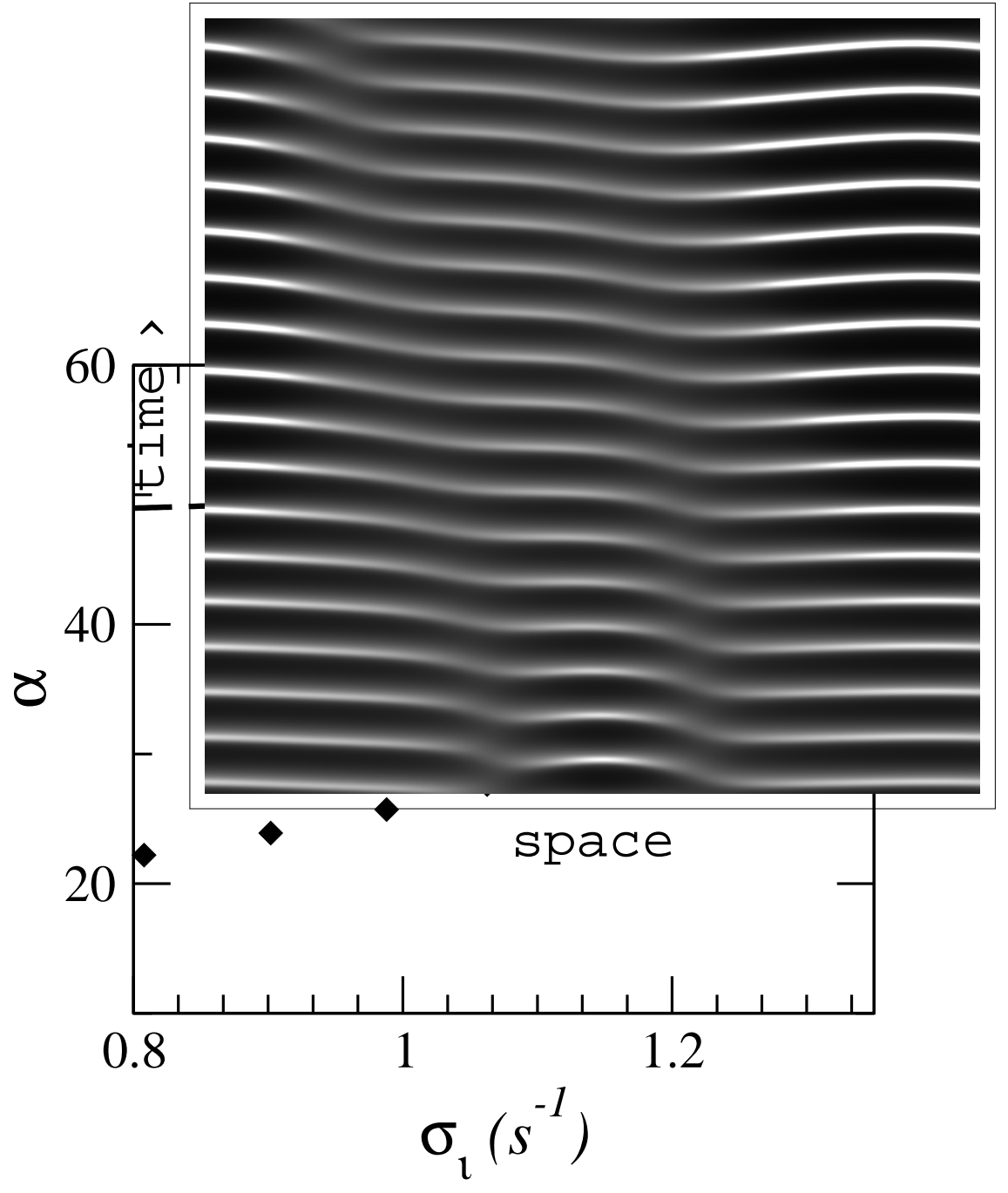




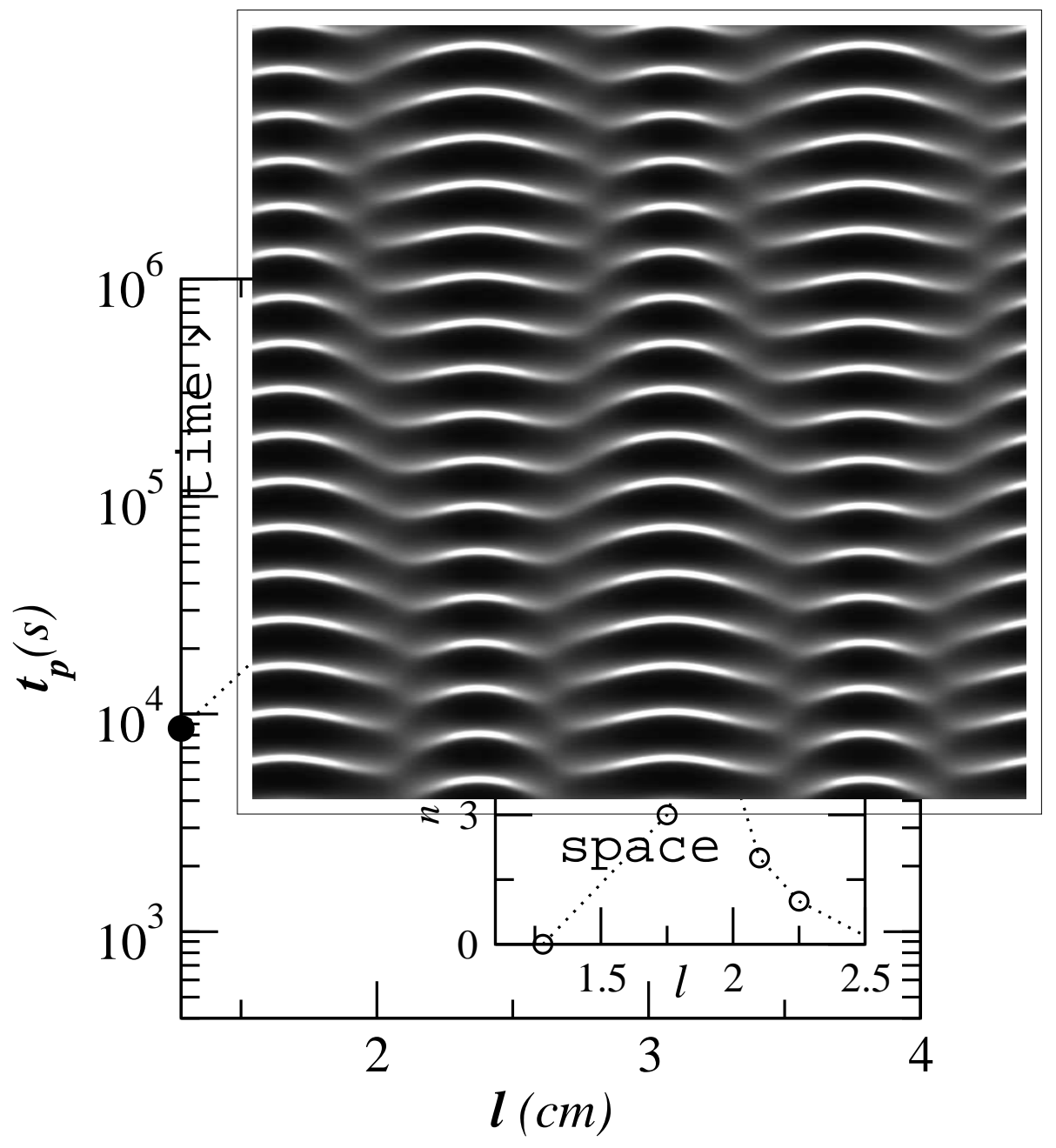




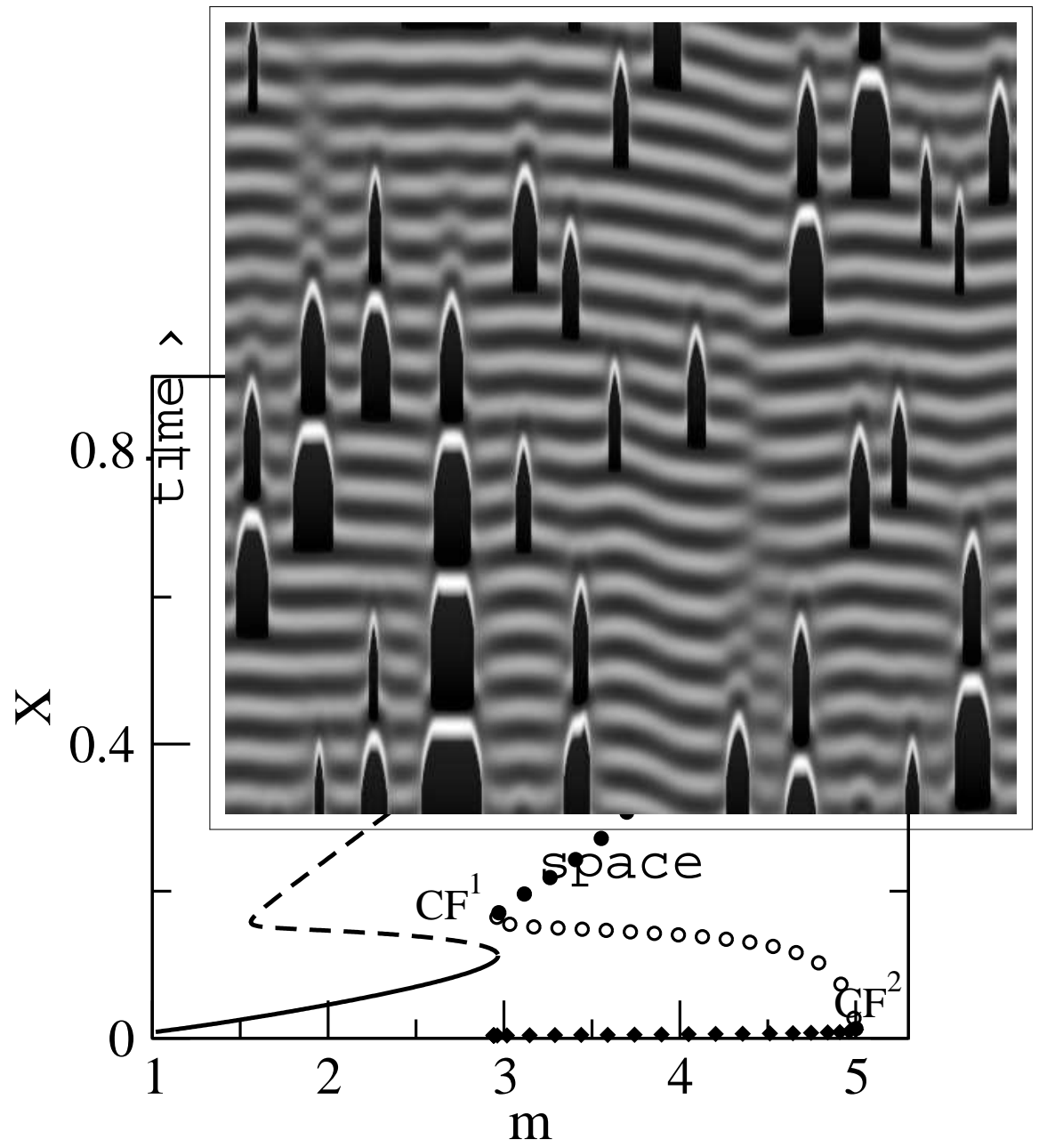

